\title{
El Recurso Agua en las Comunidades Indígenas Wayuu de La Guajira Colombiana. Parte 2: Estudio Cualitativo de las Condiciones de Higiene, Aseo y Disponibilidad de Agua
}

\author{
Alcides R. Daza-Daza ${ }^{\star}$, Ciro A. Serna-Mendoza² y Alexis Carabalí-Angola ${ }^{1}$ \\ (1) Facultad de Ingeniería, Grupo Territorios Semiáridos del Caribe, Univ. de La Guajira, km 5 vía Maicao, \\ Riohacha La Guajira-Colombia (e-mail: adaza@uniguajira.edu.co; acarabali@uniguajira.edu.co) \\ (2) Facultad de Ciencias Contables, Económicas y Administrativas, Centro de Investigaciones en Medio \\ Ambiente y Desarrollo, Univ. de Manizales, Kra 9a \# 19-03 Campo Hermoso, Manizales-Colombia \\ (e-mail: redesomciro@hotmail.com)
}

${ }^{*}$ Autor a quien debe ser dirigida la correspondencia

Recibido Feb. 12, 2018; Aceptado Abr. 19, 2018; Versión final Jun. 21, 2018, Publicado Dic. 2018

\begin{abstract}
Resumen
El estudio tuvo como finalidad analizar mediante la implementación de herramientas cualitativas de investigación las condiciones de higiene, aseo y disposición del recurso agua en las comunidades indígenas de La Guajira Colombiana. Se identificó en las zonas analizadas las concepciones que la población tiene con relación al agua y el saneamiento básico dentro de sus territorios. Las apreciaciones que la población presentó con relación a la calidad del agua estuvieron condicionadas por los factores culturales y las relaciones que estos han establecido a través de los años con su entorno natural. Los resultados muestran que los jagüeyes (zanjas) y los pozos son las principales fuentes de abastecimiento de agua que implementan la etnia Wayuu en el departamento. Se concluye que los malos hábitos higiénicos, las condiciones de aridez, la dispersión de los territorios indígenas, la carencia de sistemas de abastecimiento eficientes y la ausencia institucional son causas que inciden en el consumo de agua de mala calidad.
\end{abstract}

Palabras clave: comunidades wayuu; higiene; aseo; disponibilidad de agua

\section{The Water Resource in the Wayuu Indigenous Communities of La Guajira Colombiana. Part 2: Qualitative Study of the Conditions of Hygiene, Cleanliness and Water Availability}

\begin{abstract}
The study had the purpose of analyzing, through the implementation of qualitative research tools, the conditions of hygiene, cleanliness and water disposal resource in the indigenous communities of the Colombian guajira. The conceptions that the population has related to water and basic sanitation within their territories were identified in the analyzed zones. The appreciations of the population in relation to water quality have been conditioned by cultural factors and the relationships they have established over the years with their natural environment. The results show that jagüeyes (ditches) and wells are the main sources of water supply that implement the Wayuu ethnic group in the department. It is concluded that bad hygienic habits, living conditions, the dispersion of indigenous territories, the lack of efficient supply systems and institutional absence are the main causes that affect the consumption of water of poor quality.
\end{abstract}

Keywords: wayuu communities; hygiene; cleanliness; water availability 


\section{INTRODUCCIÓN}

El agua es reconocida como el eje central para alcanzar los objetivos del desarrollo sostenible; en este sentido, los estados deben garantizar y vigilar por su disponibilidad, sostenibilidad y el saneamiento seguro de las generaciones presentes y futuras. Igualmente, le corresponde a los países fortalecer a las poblaciones rurales en el mejoramiento de los aspectos de calidad del recurso y saneamiento (CEPAL, 2017). Así mismo, en los territorios indígenas la gestión del agua se debe abordar desde un enfoque integral que involucre los aspectos económicos, ambientales y sociales de las comunidades (Ait-Kadi, 2015). En este sentido, las comunidades indígenas han generado en torno al agua una serie de representaciones. El agua simboliza ese ser integrador que une y limita a los territorios través de los flujos de corriente que en este forman. Bajo este precepto, Jackson et al., (2013) considera que la cosmología indígena es un componente importante dentro de las culturas para conservar y manejar de forma sostenible sus recursos hídricos. Para las etnias, el agua constituye un recurso de supervivencia, es un regalo de los dioses mitológicos, es el ser que da vida a los seres de la tierra, es un recurso que se venera, respeta y se cuida (Lori et al., 2016).Por lo anterior, la disponibilidad y sostenibilidad del agua dentro de las comunidades está influenciado por el valor sistémico que estas le dan al recurso como bien ecosistémico e integral de la vida humana (Daza, 2016).

Por otra parte, en lo que corresponde a las condiciones de disponibilidad del recurso agua y saneamiento, las áreas localizadas en zonas rurales alejadas de los centros urbanos tienden a tener los menores niveles de acceso a servicios de agua potable y saneamiento. Lo anterior, se debe en parte a los problemas sociales, económicos, ambientales, políticos y culturales que enfrentan los las minorías étnicas dentro de sus territorios (Tinoco, et al., 2014). La disminución de las condiciones de calidad del agua significa para las comunidades indígenas el detrimento de la salud de sus recursos y de la vida. Por consiguiente, la sostenibilidad de los recursos ecosistémicos y de la población local se hace más compleja de alcanzar bajo estas necesidades (Sarkar et al., 2015).

Para el caso de Colombia se tiene que la mayoría de los resguardos indígenas presentes no cuentan con las condiciones básicas para atender las necesidades de saneamiento y agua potable. En esta media, las poblaciones asentadas en las zonas rurales han estado en un completo abandono producto de la centralización de los recursos económicos hacia las cabeceras municipales (Méndez et al., 2011). Estas necesidades, han ocasionado que los territorios indígenas se han más vulnerables a los riesgos causados por la ausencia de medidas sanitarias adecuadas para mejorar sus entornos de vida. Igualmente, algunas regiones del país se caracterizan por presentar déficit hídrico y problemas de acceso al recurso. En términos del agua de consumo humano estas derivan de fuentes superficiales y subterráneas las cuales en su mayoría cuentan con problemas de calidad (Borrero et al., 2013).

En lo que respecta a las comunidades indígenas Wayuu sus territorios se caracterizan por presentar escasez y deficiencias en la calidad de sus fuentes de abastecimiento agua. En este aspecto, el saneamiento básico y el acceso a un agua segura por parte de los grupos étnicos se dificultan por la dispersión de población y por los altos índices de aridez que se presentan a lo largo del territorio (Bonet et al., 2017). Con respeto al valor y percepción que tienen las comunidades indígenas Wayuu sobre el agua, se tiene todo un espacio mitológico donde el agua representa la grandeza de la coexistencia humana, sus temores, sueños, rituales y subsistencia (Chacín, 2016). El agua para las comunidades indígenas simboliza su existencia y su sostenibilidad garantiza el bienestar de las generaciones presentes y futuras (Jiménez et al., 2014; Finn y Jackson, 2011). Partiendo de lo antes expuesto, el presente trabajo pretende analizar mediante la implementación de herramientas cualitativas de investigación las condiciones de higiene, aseo y disponibilidad del recurso agua en las comunidades indígenas Wayuu de La Guajira Colombiana.

\section{MATERIALES Y MÉTODOS}

El estudio se desarrolló en 20 comunidades indígenas Wayuu de La Guajira Colombiana, utilizando la entrevista y la observación como técnicas cualitativas de investigación para la recolección y registro de la información. La descripción en detalle del procedimiento metodológico del presente artículo se describe en el estudio titulado el recurso agua en las comunidades indígenas Wayuu de La Guajira Colombiana. Parte 1: una mirada desde los saberes y prácticas ancestrales.

\section{Generalidades de la zona de estudio}

El departamento de La Guajira está habitado por negros, blancos, árabes, indígenas y criollos. De estos, las comunidades indígenas representan el $45 \%$ de la población total del departamento catalogando como uno de los territorios con mayor diversidad étnica (Cámara de Comercio de La Guajira, 2016). Las comunidades indígenas Wayuu ocupan un área de 10.080 .336 hectáreas. Habitan en un territorio donde las condiciones de baja pluviosidad y de clima árido influyen en la disponibilidad del recurso agua. El modelo de poblamiento es 
disperso, con una ocupación de espacio aproximada de 8 personas por $\mathrm{km}^{2}$ completamente retirado de los centros urbanos. (Duran, 2010).

\section{RESULTADOS Y DISCUSIÓN}

Los resultados se presentan en tres secciones; en la primera parte, se analiza desde el saber y la opinión de la población indígena Wayuu sus concepciones acerca de la calidad del recurso hídrico, en la segunda, se evalúa la disponibilidad del recurso dentro del territorio y en la última sección se aborda la problemática del saneamiento.

\section{Tratamiento y calidad del agua de consumo}

En términos generales en la actualidad las comunidades indígenas Wayuu residentes en la Alta y Media Guajira no tienen costumbre de realizar algún tipo de tratamiento al agua utilizada para consumo humano. En este aspecto, las comunidades relacionan que muchas de las enfermedades presentes en sus territorios como la diarrea y las infecciones en la piel son por causa del uso de agua de mala calidad. En este sentido, se tiene que al igual que en las comunidades de La Guajira, existen a nivel mundial experiencias investigativas que argumentan que los grupos étnicos al ser sectores que no cuentan con las condiciones de agua potable y saneamiento básico adecuadas, son considerados como zonas propensas al contagio de este tipo de enfermedades; como ejemplo claro, se tienen las poblaciones indígenas de Ata Manobos, Dibabawons, Mandayas y Mansakas localizadas en la región Davao al sur de Filipinas, donde los grupos indígenas presentan problemas de salud derivados de los malos hábitos higiénicos (Belizario et al., 2011). Cabe mencionar además, que las comunidades son conscientes de que el agua que toman no cumplen con los criterios mínimos de calidad; sin embargo, su consumo se sigue presentando. Estudios realizados en las comunidades étnicas de Canadá revelan que uno de los mayores factores de riesgos de contaminación de las fuentes de agua provienen de la manipulación inadecuada de las heces fecales, causantes de enfermedades gastrointestinales, igualmente, consideran que existen vacíos de información relacionados con el tipo de agua que consumen los infantes y las enfermedades trasmitidas por el agua (Bradford et al., 2016). En este punto, es necesario aclarar que en el proceso de cocción de muchos alimentos y bebidas que tienen las comunidades Wayuu dentro de su dieta, permiten que de cierta forma el agua pase por un proceso de desinfección. Para el caso de las zonas estudiadas, las comunidades cuentan con fuentes de abastecimiento como jagüeyes, pozos, casimbas, albercas, carrotanques, represas y plantas desalinizadoras que las proveen del preciado líquido (Figura 1).

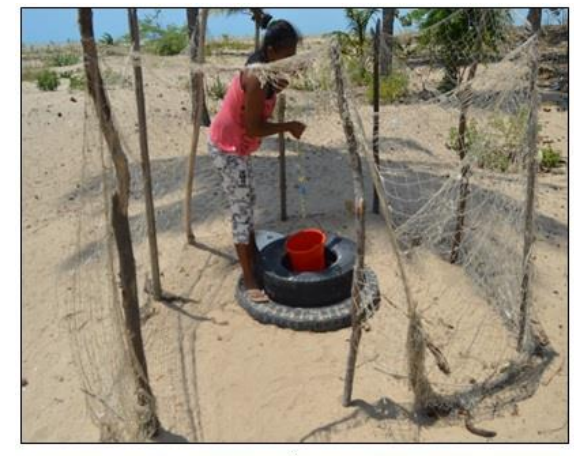

a)

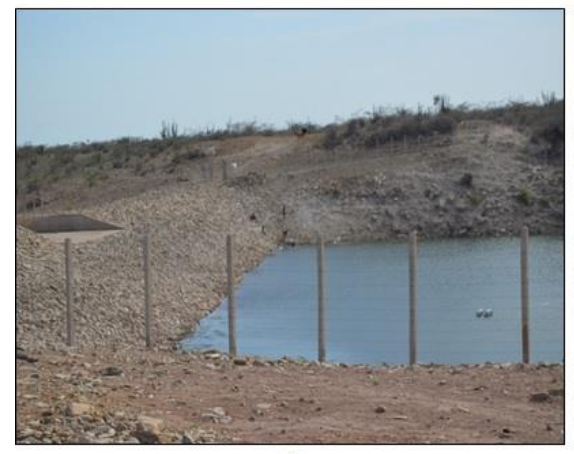

d)

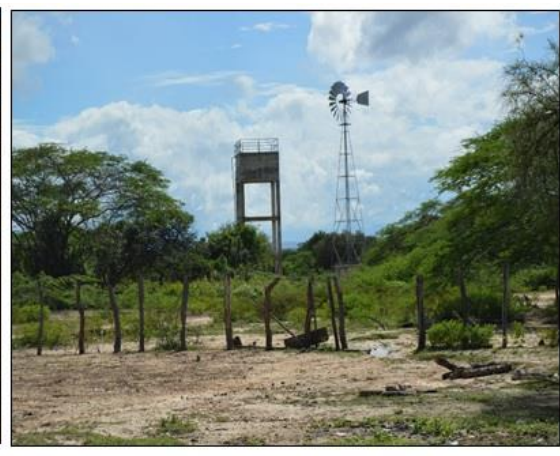

b)

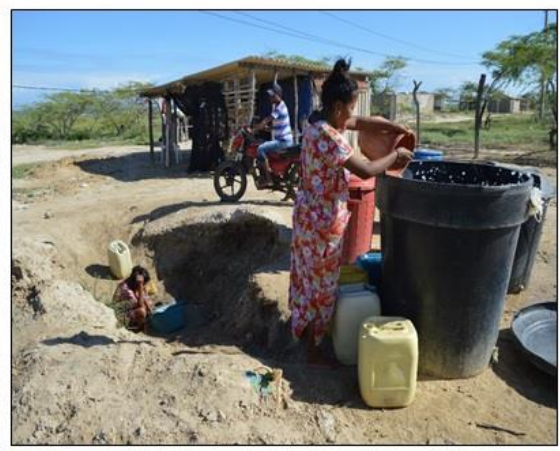

e)

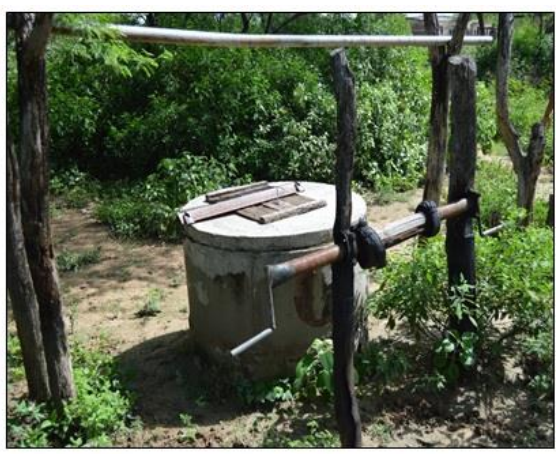

c)

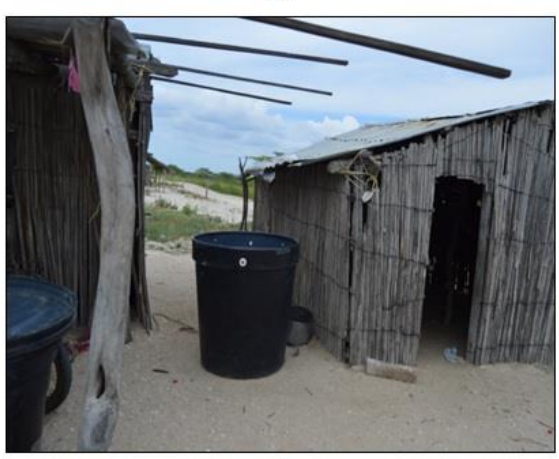

f)

Fig. 1: Sistemas de captación y abastecimiento de agua: a) Pozos artesanales (Comunidad Los Cocos); b) Captación mediante el uso de molinos de viento (Comunidad Guacharaquero); c) Pozo manual (Comunidad Guacharaquero); d) Represa de agua (Comunidad Wososopo); e) Almacenamiento de agua en tanques (Comunidad kouchachon); y $\mathrm{f}$ ) Captación de agua lluvia (Comunidad Carrizal) 
En cuanto a lo expresado anteriormente, se hace necesario educar a la población sobre la importancia de tratar el agua y los beneficios que esto trae para reducir las enfermedades intestinales. Igualmente, es indispensable que las comunidades con el apoyo de los gobiernos locales implementen estrategias de tratamiento, protección y monitoreo de la calidad de las fuentes de agua (Rowles et al., 2018). Por su parte, Wright (2018) y Alam (2017) consideran que las percepciones que las comunidades pueden tener alrededor de la calidad del agua están condicionadas por los factores culturales y las relaciones que estas han establecido a través de los años con su entorno natural. De esta manera, las poblaciones han conservado y aprovechado sus recursos de forma sostenible. Sin embargo, las condiciones climáticas y los procesos de desarrollo industrial y económico presentados en los territorios han atentado contra la calidad y disponibilidad de los mismos.

En este sentido, las entrevistas realizadas a lo largo de las áreas de estudios dejan entrever las razones por las cuales las comunidades consumen sin ningún tipo de tratamiento el agua. Dentro de estas, se encuentra la dispersión de los territorios indígenas, la lejanía de las fuentes de abastecimiento, el mal estado de las vías, la carencia de sistemas de abastecimiento eficientes y la ausencia de un órgano de gestión del recurso hídrico por parte del gobierno departamental que garantice a las comunidades la presencia de agua potable. Conviene subrayar que muchas veces las condiciones agrestes de aridez y escasez de agua obligan al Wayuu a consumir agua de mala calidad con tal de satisfacer su necesidad producida por la sed. A causa de esto, los Wayuu acarrean agua de los jagüeyes, casimbas y pozos de calidad no óptima para el consumo humano. Asimismo, la emplean para bañarse, preparar sus alimentos y abastecer sus cultivos y animales sin ningún control sanitario. De igual forma, a pesar que ancestralmente no es costumbre del Wayuu realizar prácticas para hervir el agua; si era tradición en algunas comunidades la implementación de acciones para su purificación, como es el caso del uso de paños para filtrarla, dejarla en reposo o implementar técnicas con la pulpa del cactus para sedimentar las partículas sólidos. En este sentido es claro ver como las comunidades con el pasar del tiempo han perdido sus saberes y practicas ancestrales.

Se debe agregar además, que para el Wayuu el agua de lluvia es concebida como el agua de buena calidad y es utilizada exclusivamente para beber. El agua proveniente de los pozos fue catalogada por la población como salobre, la de los jagüeyes como turbia y la adquirida de los carrostanques por presentar olor a cloro. De acuerdo con Anderson et al. (2014) el concepto de calidad del agua por parte de las comunidades indígenas está íntimamente relacionado con el bienestar del entorno natural que les rodea; de esta forma, se garantiza que el ser humano goce de salud durante su existencia en el mundo terrenal.

\section{Disponibilidad y acceso al recurso Agua}

Las insuficiencias en el acceso al agua segura en el departamento de La Guajira están marcado por la baja oferta hídrica y las deficiencias institucionales en los aspectos de gestión de los recursos hídricos. Sumado a esto, se tiene que la escasez de la disponibilidad de agua en las regiones de la Alta y Media Guajira está sujetada a la capacidad de almacenamiento que puedan tener los pozos y reservorios y a la dependencia de aprovisionamiento de agua por parte de los entes municipales y departamentales (Cantillo et al., 2016). Partiendo de lo anterior, en las comunidades visitadas, se encontró que la población debe realizar largos recorridos para poder abastecerse de agua; en este caso, las fuentes de abastecimiento de mayor uso son los jagüeyes y pozos, los cuales se encuentran en su mayoría a más de $1 \mathrm{~km}$ de distancia. Igualmente, es de resaltar que la disponibilidad de agua que pueda presentar un reservorio está sujeta a la capacidad que este tenga para resistir a los efectos de la radiación solar, del viento y la infiltración. Producto de esto, las comunidades se ven obligadas a desplazarse de un sector a otro en busca de nuevas fuentes de abastecimiento que les permitan proveerse del recurso; lo que termina, incidiendo con las distancias y el tiempo que estos emplean en el desarrollo de esta actividad. Por lo tanto, es necesario destacar que en términos generales los reservorios duran con agua aproximadamente entre 4 a 6 meses a lo largo de un año.

Cabe señalar que en términos de conservar la disponibilidad de agua en las fuentes de abastecimiento, cada familia emplea estrategias para disminuir la cantidad de agua utilizada durante el baño, como son el uso de agua de mar y el humedecimiento paños con el fin de asear el cuerpo. Por otra parte, aunque actualmente las prácticas ancestrales se han perdido, las comunidades Wayuu para sosegar la sed de sus animales les suministraban la pulpa del Yosú (Stenocereus griseus) como fuente de suministro de agua. Igualmente, esta especie vegetal se empleaba en la clarificación de las aguas turbias (Villalobos et al., 2007). Hay que mencionar además, que cuando la disponibilidad del agua es mínima, las actividades físicas por parte de la comunidad son limitadas con el fin de que el cuerpo no demande el consumo excesivo de agua. La población de la Alta Guajira cercanas al mar utilizan la técnica de pozos artesanales (excavan hasta llegar a la arena húmeda y esperan que esta se llene por infiltración) para obtener agua dulce. De igual forma, el agua de mar es utilizada en las diferentes labores de la vida cotidiana de los Wayuu. En relación con esto, Black y McBean (2017) reconocen la importancia de incluir los saberes ancestrales en los procesos de gestión del recurso hídrico por parte de los órganos tomadores de decisiones, con el fin de garantizar su sostenibilidad. 


\section{Actividades de Higiene y aseo de las comunidades indígenas Wayuu}

Los resultados de las entrevistas muestran que las actividades de baño y lavado de la ropa no se realizan directamente en las fuentes de agua; sino, que se desarrollan a una distancia prudente del reservorio. En época de lluvia las personas se asean hasta 3 veces al día; mientras, que en el periodo de verano la población acostumbra a bañarse una sola vez al día y generalmente esta actividad se hace en horas de la mañana o en las horas de la tarde, conservando una distancia prudente entre la zona de baño y la fuente de agua. Igualmente, el agua que se usa para el lavado de los utensilios de cocina se cambia periódicamente. Por otra parte, la población manifestó que los animales son llevados a los jagüeyes donde consumen el agua de forma directa. También se encontró que algunas comunidades ejecutan acciones de limpieza de los tanques de almacenamiento (Figura 2) y las fuentes de abastecimiento de agua. En lo referente a las viviendas los resultados de las entrevistas muestran opiniones donde la población declara que los recipientes que implementan para el almacenamiento y extracción de agua son tapados y protegidos de los contaminantes. A pesar de esto, es necesario dejar claro que no todas las comunidades indígenas Wayuu localizadas a lo largo del territorio Guajiro implementan este tipo de prácticas; en este sentido, existen territorios donde las fuentes de agua (Jagüeyes) son sometidas a diferentes usos (baño, lavado de ropa, consumo de agua, como abrevadero de los animales entre otros) sin ningún tipo de medida, quedando expuestas a todo tipo de problemas de contaminación (Figura 3).

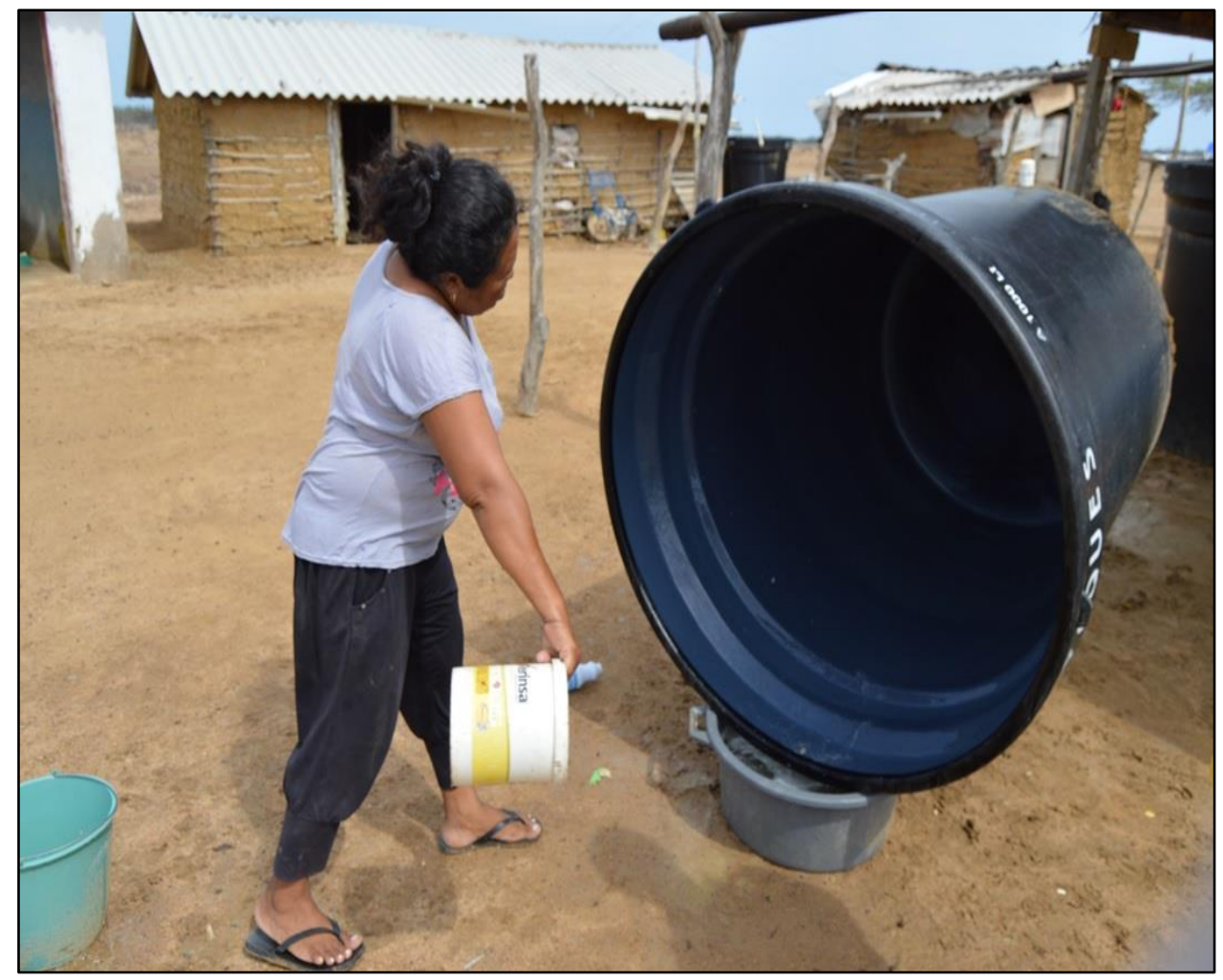

Fig. 2: Limpieza de tanque de almacenamiento de agua

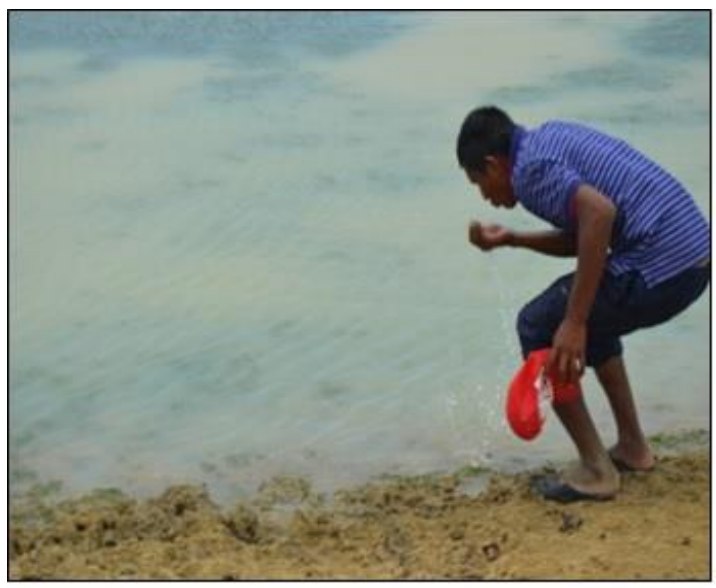

a)

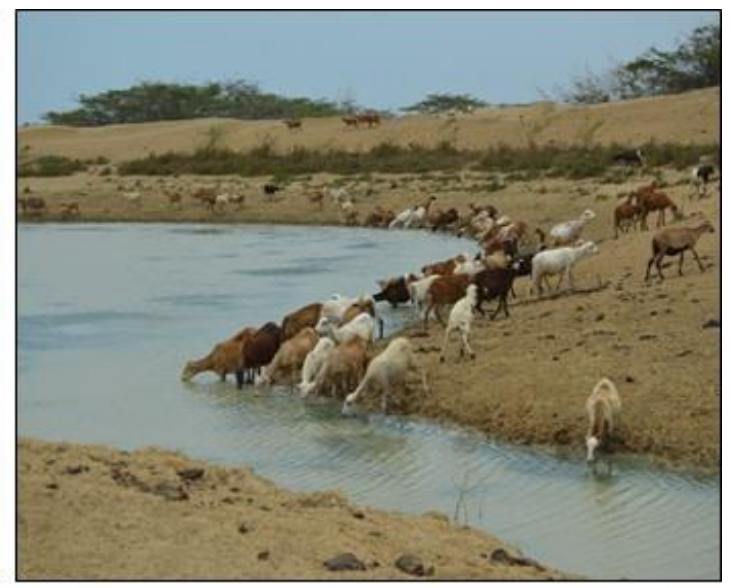

b)

Fig. 3: Uso de los jagüeyes en la comunidad de Topia: a) Wayuu consumiendo agua; y b) Animales tomando agua 
En lo que respecta a las necesidades fisiológicas, se encontró que estas son ejecutadas a campo abierto; el Wayuu en términos generales no es muy dado al uso del papel higiénico, ancestralmente la limpieza de su parte intima la realizaban empleando hojas de Bastardía Viscosa (Kashushira: plantas de hojas anchas y suaves, empleada también para quitar la grasa de las manos y de los recipientes), la tuza del maíz y palitos de ramas de los árboles. Así mismo, las comunidades manifestaron que acostumbran en algunas ocasiones a lavarse las manos antes y después de defecar y orinar. Por otra parte, las necesidades fisiológicas son realizadas cerca de las viviendas y alejadas de los cuerpos de agua. Para el caso de los residuos que generan como son las bolsas plásticas y los restos de los empaques de alimentos, en su mayoría son arrojados al suelo, otras veces son enterrados y en ocasiones incinerados. Los desperdicios de los alimentos son dados a los animales domésticos (Figura 4).

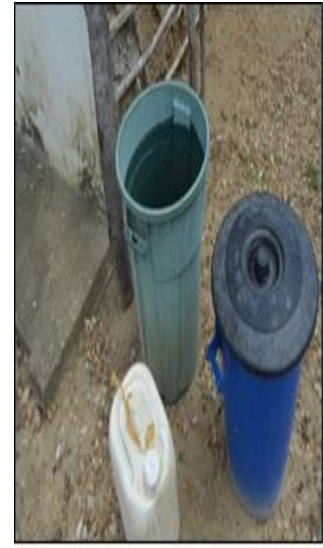

a)

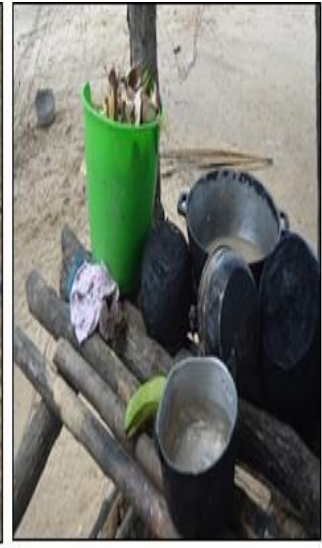

b)

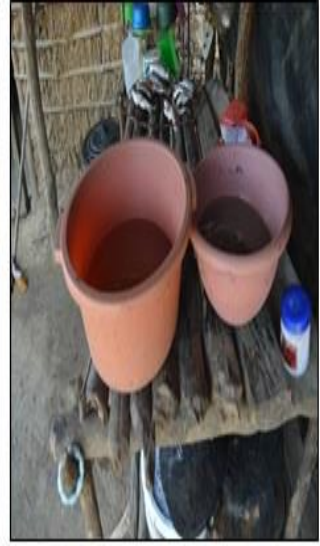

c)

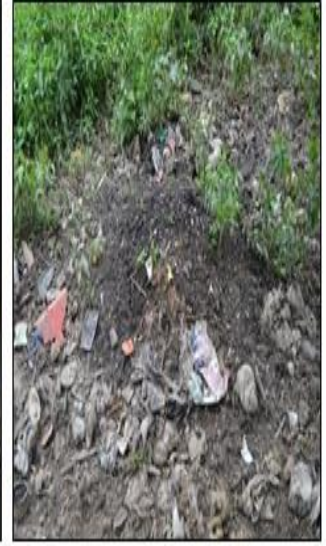

d)

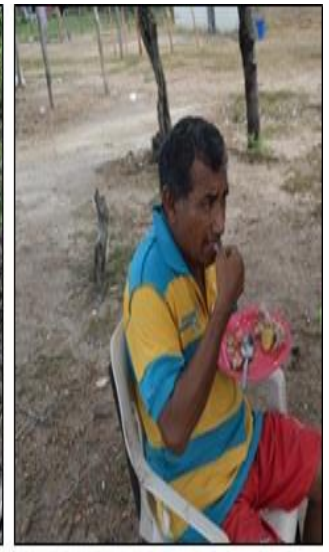

e)

Fig. 4: Generación de residuos, manejo y uso del agua: a) Recipientes sin tapas; b) Almacenamiento de residuos de comunidad; c) Almacenamiento de agua para lavar los utensilios de cocina; d) Incineración de residuos; e) Consumo de alimentos

Conviene decir que alrededor del saneamiento y las necesidades fisiológicas realizadas al aire libre existen a lo largo de la historia innumerables concepciones por parte de los grupos sociales. En el caso de las comunidades indígenas de La Guajira Colombia, se encontró que para algunos miembros de la etnia, la eliminación de desechos corporales a campo abierto se convierte en una fuente orgánica de alimento para los animales domésticos que estos crían; como es el caso de los cerdos; además, consideran que los residuos del cuerpo se descomponen de forma natural y son fuente de nutrimiento del suelo. Igualmente, se pudo evidenciar que son conscientes que el esparcido de este tipo residuos de origen animal y humano afecta la calidad sanitaria de las aguas, generando la formación de vectores que atentan contra la salud de la población. Por otra parte, para algunas culturas de la India asentadas en las zonas rurales consideran que la realización de las defecaciones al aire libre tiene una connotación simbólica para el cuerpo. Suponen que mediante este acto se libera y purifica el ser humano. Para otras culturas asentadas en el este de Zambia contemplan que el uso de letrinas no puede ser compartido entre todos los miembros de un grupo familiar; asimismo, en la región norte de la India las comunidades a pesar de contar con letrinas domésticas, las necesidades fisiológicas las realizan a campo abierto, por considerar que de esta manera es posible disfrutar de un mejor entorno natural. De esta forma, se ve claramente como los ambientes sociales establecen sus normas y pensamientos con relación al saneamiento y la defecación (McMichael, 2017; Routray, et al., 2015; Desait, et al., 2015 y Thys et al., 2015). Por su parte, McDonald et al. (2015) considera que las barreras culturales, sociales, económicas y físicas que presentan las comunidades localizadas en las áreas rurales influyen y dificultan la implementación de programas encaminados a la promoción de conductas de higiene segura en la población. En este sentido, Rahman et al. (2017) en estudios realizados en las comunidades rurales de Bangladesh, coincide en afirmar que los bajos ingresos de la población y los altos índices de analfabetismo imposibilita la compra de agua potable por parte de las comunidades, estando más susceptibles al consumo de un agua no segura para la salud humana.

\section{CONCLUSIONES}

De acuerdo a los resultados y análisis de la investigación se pueden extraer las siguientes tres conclusiones principales: 1) La dispersión de los territorios indígenas, la lejanía de las fuentes de abastecimiento, el mal estado de las vías, la carencia de sistemas de abastecimiento eficientes y la ausencia institucional son factores que inciden en el consumo de agua de mala calidad por parte de las comunidades indígenas Wayuu; 2) La pérdida de las prácticas ancestrales alrededor del agua debilitan los valores culturales, afectando la disponibilidad de las fuentes de abastecimiento; y 3) Los hábitos de aseo e higiene presentes en la población Wayuu afectan el bienestar y la calidad de vida de población. 


\section{REFERENCIAS}

Ait-Kadi, M., Water for Development and Development for Water: Realizing the Sustainable Development Goals (SDGs) Vision, doi: 10.1016/j.aqpro.2016.06.013, Aquatic Procedia, 6(1), 106-110 (2016)

Alam, M.Z., L. Carpenter-Boggs y 6 other authors, Water Quality and Resident Perceptions of Declining Ecosystem Services at Shitalakka Wetland in Narayanganj City, doi: 10.1016/j.swaqe.2017.03.002, Sustainability of Water Quality and Ecology, 9(10), 53-66 (2017)

Anderson, K., B. Clow y M. Haworth-Brockman, Carriers of water: Aboriginal women's experiences, relationships, and reflections, https://doi.org/10.1016/j.jclepro.2011.10.023, Journal of Cleaner Production, 60, 11-17 (2014)

Belizario, J.R., F.I.G. Totañes, W.N. de Leon, Y.F. Lumampao y R.N.T. Ciro, Soil-transmitted Helminth anda otros Intestinal Parasitic Infections among School Children in Indigenous People Communities in Davao the Norte Philippines, doi: doi.org/10.1016/j.actatropica.2011.02.010, Acta Tropical, 120, 12-18 (2011)

Black, K. y E. McBean, First Nations' Water Sustainability and Security Strategy: Tools and Methodologies for CommunityDriven Processes for Water Treatment in Indigenous Communities, doi: 10.1016/j.techsoc.2017.04.004, Technology in Society, 50, 57-65 (2017)

Bradford, L.E., L.A. Bharadwaj, U. Okpalauwaekwe y C.L. Waldner, Drinking Water Quality in Indigenous Communities in Canada and Health Outcomes: a Scoping Review, doi: 10.3402/ijch.v75.32336, International Journal of Circumpolar Health, 75(1), 32336 (2016)

Bonet, J. y L.W. Hahn, La Mortalidad Infantil en La Guajira: Una Caracterización Estructural, Banco de la República sucursal Cartagena, ISSN: 1692-3715, 255(1), 1-63 (2017)

Borrero, J.L., F. Montoya y L.A. Escobar, Desarrollo de un Sistema Aerodesalinizador para la Potabilización de Acuíferos Salobres en La Guajira Colombiana, doi: 10.21774/ing.v7i17.312, Ingenius, 7(17), 11-18 (2013)

Cantillo, K.C., G.A. Vargas, N.A. Sakzuk, D. Díaz, E.D. Vallejo y C. Castalleda, Hambre y Desnutrición en La Guajira, Instituto Nacional de Salud (2016)

Chacín, H., Asombros del Pueblo Wayuu, 1aㅡ Ed., 1-150, Fondo editorial Unermb, Maracaibo, Venezuela (2016)

CDC, Cámara de Comercio de La Guajira, Informe Socioeconómico del Departamento de La Guajira 2016, CDC (2016)

CEPAL, NU. The 2030 Agenda and the Sustainable Development Goals: An Opportunity for Latin America and the Caribbean, United Nations publication, Santiago, Chile (2017)

Daza, A.R., La Crisis del Agua en La Guajira: un Análisis Crítico Frente al Concepto del Desarrollo Sostenible, Revista Asuntos Económicos y Administrativos, 31(1), 151-160 (2016)

Desai, R., C. McFarlene y S. Graham, The Politics of Open Defecation: Informality, Body, and Infrastructure in Mumbai, doi: 10.1111/anti.12117, Antipode, 47(1), 98-120 (2015)

Duran, V.H., Cuerpo y Educación en la Cultura Wayuu, Revista Educación Física y Deporte, 29(2), $239-252$ (2010)

Finn, M. y S. Jackson, Protecting Indigenous Values in Water Management: a Challengue Conventional Environmental Flow Assessments, doi: 10.1007/s10021-011-9476-0, Ecosystems, 14(1), 1232-1248 (2011)

Jackson, S. y M. Barber, Recognition of Indigenous Water Values in Australia's Northern Territory: Current Progress and Ongoing Challenges for Social Justice in Water Planning, doi: 10.1080/14649357.2013.845684, Planning Theory y Practice, 14(4), 435-454 (2013)

Jiménez, A., M. Cortobius y M. Kjellén, Trabajando con Pueblos Indígenas en Agua y Saneamiento Rural: Recomendaciones para un Enfoque Intercultural, Stockholm International Water Institute, SIWI (2014)

Lori, E. A., L.A. Bradford, L.A. Bharadwaj, O. Udoka y L.W. Cheryl, Drinking Water Quality in Indigenous Communities in Canada and Health Outcomes: a Scoping Review, doi: 10.3402/ijch.v75.32336, International Journal of Circumpolar Health, 75(1), 2242-3982 (2016)

Méndez, F.S., M. Opazo, P. Romero y B.C. Pérez, Metodología para la Apropiación de Tecnologías de Saneamiento Básico en Comunidades Indígenas, Cuaderno de desarrollo rural, ISSN: 0122-1450, 8(66), 153-176 (2011)

Mcdonald, E., T. Cunningam y N. Slavin, Evaluating a Handwashing with Soap Program in Australian Remote Aboriginal Communities: a pre and post Intervention Study Design, doi: 10.1186/s12889-015-2503-x, BMC public health, 15(1), 1188 (2015)

McMichael, C., Toilet Talk: Eliminating Open Defecation and Improved Sanitation in Nepal, doi: 10.1080/01459740.2017.1371150, Medical Anthropology, 1-17 (2017)

Rahman, M.T.U., M. Rasheduzzaman, M.A. Habib, A. Ahmed, S.M. Tareq, y S.M. Muniruzzaman, Assessment of Fresh Water Security in Coastal Bangladesh: an Insight from Salinity, Community Perception and Adaptation, doi: 10.1016/j.ocecoaman.2016.12.005, Ocean y CoastalManagement, 137(1), 68-81 (2017)

Routray, P., W.P. Schmidt, S. Boisson, T. Clasen y M.W. Jenkins, Socio-Cultural and Behavioural Factors Constraining Latrine Adoption in Rural Coastal Odisha: an Exploratory Qualitative Study, doi: 10.1186/s12889-015-2206-3, BMC Public Health, 15(1), 880 (2015) 
Rowles, L.S., R. Alcald y 12 other authors, Perceived Versus Actual Water Quality: Community Studies in Rural Oaxaca, México, doi: 10.1016/j.scitotenv.2017.11.309, Science of the Total Environment, 622, 626-634 (2018)

Sarkar, A., M. Hanrahan y A. Hudson, Water Insecurity in Canadian Indigenous Communities: some Inconvenient Truths, Rural and Remote Health, ISSN: 1445-6354, 15(3354), 1-14 (2015)

Tinoco, M., M. Cortobius, M. Doughty y M. Kjellén, Water Co-Operation between Cultures: Partherships with Indigenous Peoples for Sustainable Water and Sanitation Services, doi: 10.1016/j.aqpro.2014.07.009, Aquatic Procedia, 2(1), 55-62 (2014)

Thys, S. y K.E. Mwape, Why Latrines are not Used: Communities' Perceptions and Practices Regarding Latrines in a Taenia Solium Endemic Rural Area in Eastern Zambia, doi: 10.1371/journal.pntd.0003570, PLOS Neglected Tropical Diseases, 9(3), e0003570 (2015)

Villalobos, S., O. Vargas y S. Melo, Uso, Manejo y Conservación de "Yosú", Stenocereus Griseus (Cactaceae), en la Alta Guajira Colombiana, Acta biológica Colombiana, ISSN: 0120-548X, 12(1), 99-112 (2007)

Wright, C.J., J.M. Sargeant y 8 other authors, How are Perceptions Associated with Water Consumption in Canadian Inuit? A Cross-Sectional Survey in Rigolet, Labrador, doi: 10.1016/j.scitotenv.2017.10.255, Science of The Total Environment, 618, 369-378 (2018) 